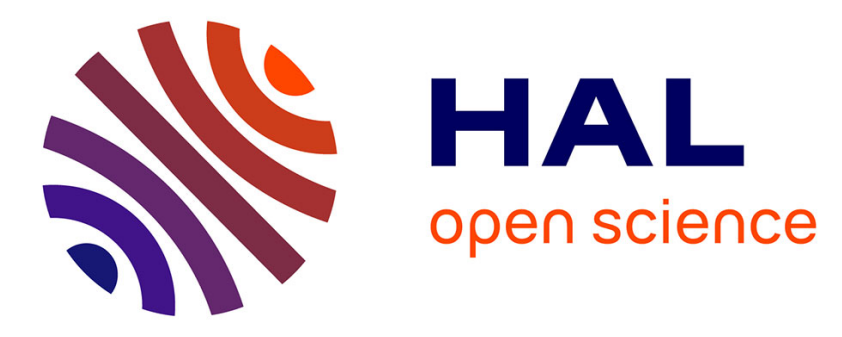

\title{
Les entreprises peuvent-elles faire confiance à la confiance?
}

\author{
Anne-Claire Chêne, Joan Le Goff
}

\section{To cite this version:}

Anne-Claire Chêne, Joan Le Goff. Les entreprises peuvent-elles faire confiance à la confiance?: Une exploration du lien contrôle-confiance comme principe organisationnel. Revue internationale de psychosociologie et de gestion des comportements organisationnels, 2017, L'entreprise libérée, XXIII (56), pp.185-204. hal-01677967

\section{HAL Id: hal-01677967 https://hal.science/hal-01677967}

Submitted on 8 Jan 2018

HAL is a multi-disciplinary open access archive for the deposit and dissemination of scientific research documents, whether they are published or not. The documents may come from teaching and research institutions in France or abroad, or from public or private research centers.
L'archive ouverte pluridisciplinaire HAL, est destinée au dépôt et à la diffusion de documents scientifiques de niveau recherche, publiés ou non, émanant des établissements d'enseignement et de recherche français ou étrangers, des laboratoires publics ou privés. 


\title{
LES ENTREPRISES PEUVENT-ELLES \\ FAIRE CONFIANCE A LA CONFIANCE ? \\ Une exploration du lien contrôle-confiance \\ comme principe organisationnel
}

\author{
Anne-Claire Chêne $e^{1}$ Joan Le Goff
}

\section{RESUME}

Utiliser des contrôles pour développer la confiance au sein des organisations? Impossible ! Et pourtant... Cette recherche menée auprès de salariés et managers d'une organisation ayant fait le choix de la confiance comme principe organisationnel invite à envisager comment des contrôles permettent de développer non pas une confiance paradoxale mais une confiance assurée. Celle-ci requiert la combinaison d'une vision positive de l'individu et d'une volonté d'intégrer le risque au lieu de le maîtriser, permettant d'utiliser les contrôles de manière coopérative dans un intérêt commun.

${ }^{1}$ Doctorante, IRG (EA 2354) Université Paris Est Créteil, ac.chene@gmail.com

${ }^{2}$ Professeur en sciences de gestion, Joan Le Goff dirige l'UFR de sciences économiques et de gestion de l'université Paris-Est Créteil et conduit ses recherches au sein de l'IRG, legoff@u-pec.fr 


\section{LES ENTREPRISES PEUVENT-ELLES FAIRE}

CONFIANCE A LA CONFIANCE ?

.1

Anne-Claire Chêne, Joan Le Goff

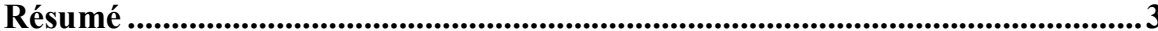

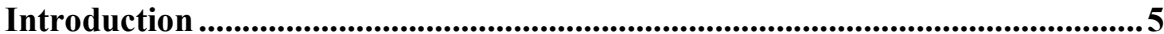

1. Contrôle vs confiance, ou contrôle et confiance ? ........................................... 7

1.1 Entre confiance impossible et confiance idéale..................................... 7

1.2 Entre contrôles formels et sociaux coercitifs et contrôles coopératifs ... 10

1.3 Contrôles coopératifs et confiance idéale : une supplémentation

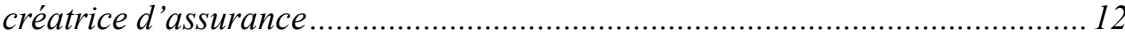

2. Étude de cas : la confiance comme principe organisationnel .....................14

2.1 Choix d'une équipe de l'entreprise Hervé Thermique.......................... 14

2.2 De la confiance idéale à la confiance assurée ….................................. 17

2.3 Mise en cuvre des contrôles coopératifs............................................ 18

Conclusion : une façon d'envisager l'organisation et les relations ...................20

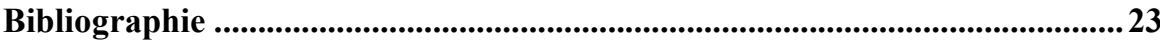




\section{INTRODUCTION}

Et la confiance, bordel ? Ce titre d'un ouvrage publié par l'Institut Montaigne en 2014 atteste l'enjeu que représente la confiance pour les entreprises, non seulement pour le bien-être des salariés mais aussi pour l'efficacité du travail et la compétitivité des organisations. Il montre aussi que les organisations ne savent pas forcément comment faire pour la mettre en œuvre et qu'elles ont tendance à avoir recours aux normes, aux règles et procédures, donc à renforcer les contrôles coercitifs, dans un contexte où la confiance serait plus pertinente. Le fonctionnement fondé sur le contrôle directif était approprié lorsque les organisations voulaient atteindre un résultat prévisible et facilement mesurable. En revanche, un fonctionnement basé sur la confiance est beaucoup plus efficace dans les situations demandant adaptabilité, créativité, agilité. La confiance est par exemple nécessaire pour activer la motivation, la citoyenneté organisationnelle, la satisfaction au travail, l'implication, la prise de risque, la coopération et la poursuite d'un objectif commun, leviers de performance dans l'environnement actuel (Gratacap et al., 2011).

Initialement, la confiance était perçue comme irrationnelle en sciences de gestion. Elle a été mobilisée en faisant appel à la psychologie et à la sociologie pour modérer la vision rationaliste et prendre en compte le fait que les individus ont des affects et sont influencés par le contexte dans lequel ils évoluent. L'emploi du contrôle et le recours à la confiance ont été présentés comme antagonistes, comme deux visions des relations et organisations que tout oppose ou qui peuvent se substituer l'une à l'autre. D'abord considérée par dépit lorsque le contrôle formel était défaillant ou insuffisant, la confiance a ensuite été considérée comme un choix facteur d'efficience. La complémentarité entre confiance et contrôle a alors été étudiée, faisant ressortir son usage dans l'objectif de servir un meilleur contrôle, dans une perspective utilitariste. Si la confiance est employée comme mécanisme de manipulation, de domination, de contrainte (Bornarel, 2004), et si la perte de contrôle fait peur, il n'est pas surprenant de constater que les managers n'ont pas consacré suffisamment de temps, d'énergie et de ressources à créer la confiance dans les organisations. Peut-être les managers n'ont-ils pas envie d'avoir recours à la confiance, ou peut-être n'ont-ils pas envie d'avoir recours à la confiance dans ces conditions-là.

Des entreprises qui se disent aujourd'hui libérées ou en cours de libération revendiquent la confiance comme moteur de leur fonctionnement. Est-il possible pour une organisation d'employer la confiance comme principe organisationnel ? Dans ce cas, la confiance remplace-t-elle le contrôle ou vient-elle s'y combiner d'une nouvelle manière ? Cette question nous invite à renverser l'articulation entre confiance et contrôle et à envisager que des contrôles puissent servir la confiance, si ces deux notions ne sont pas antagonistes. 
Dans cette perspective, nous avons développé un cadre conceptuel autour des notions de confiance et contrôle et de leur articulation (partie 1). Nous l'avons confronté à une étude exploratoire qualitative qui a fait émerger du terrain des modalités selon lesquelles des mécanismes de contrôle peuvent contribuer à développer la confiance dans un contexte organisationnel (partie 2). Nous finirons par les limites de cette étude et des pistes complémentaires de recherche.

\section{CONTROLE VS CONFIANCE, OU CONTROLE ET CONFIANCE ?}

\subsection{Entre confiance impossible et confiance idéale}

L'approche économique classique et les théories contractuelles reposent sur une vision de l'individu opportuniste, aliéné à ses intérêts, et sur les hypothèses fondamentales de divergences d'intérêt et de maximisation du profit. Dans ce contexte, l'organisation cherche à supprimer le risque a priori pour garantir l'atteinte des objectifs organisationnels et voit le contrôle comme le moyen d'y parvenir. L'organisation scientifique du travail, développée à une époque où l'environnement était relativement stable, considère que la confiance est irrationnelle et vise à supprimer toute interférence et tout facteur générant de l'incertitude, notamment toute relation interpersonnelle. Dans ce cas, la confiance est impossible (Bornarel, 2007) et confiance et contrôle sont antagonistes.

La confiance est apparue lorsque la psychologie et la sociologie ont été convoquées et ont fait valoir une possible vision positive de l'individu. La confiance se fonde alors sur un présupposé d'honnêteté et d'acceptation d'une obligation morale, en plus de la croyance que l'individu a les compétences nécessaires à la réalisation de l'action. Les définitions de la confiance prennent en compte des éléments affectifs et émotionnels en plus des éléments rationnels. Dans ce cas, le risque est une composante essentielle de la confiance qui se traduit par l'acceptation par X de se rendre vulnérable vis-à-vis de $\mathrm{Y}$ parce que $\mathrm{X}$ croit que $\mathrm{Y}$ a l'état d'esprit et les compétences nécessaires pour que le niveau de risque soit considéré comme acceptable. Il s'agit d'une confiance dans laquelle se mêlent espoir et doute. Nous l'appellerons confiance-espoir. L'attitude spontanée sur laquelle s'appuie cette confiance peut être augmentée par la volonté. Lorsque cette vision positive de l'individu se combine avec un niveau de risque acceptable très faible de la part de l'organisation, on se retrouve dans un cas de confiance irréductible : l'organisation fait confiance à ses salariés pour adhérer aux principes et obéir aux règles de l'organisation et se placer dans un rapport de domination parce qu'ils trouvent leur intérêt à obéir. Cela permet en effet de réduire les risques notamment en limitant l'arbitraire à travers des procédures équitables, cas typique de l'organisation bureaucratique (Bornarel, 2007).

Ces dernières décennies, l'internationalisation de la compétition, l'intensification du rythme des innovations technologiques, la fragmentation des marchés, l'exigence 
accrue des clients et l'accélération globale du fonctionnement du marché ont mis les organisations face à un besoin croissant de rapidité et de flexibilité. Dans cet environnement de plus en plus concurrentiel et international, les organisations ont été contraintes de développer des approches permettant de faire face à une complexité et une incertitude accrues. La confiance est apparue comme un moyen de se rassurer face au risque, et ce de deux manières principales. La première consiste à tout miser sur la vision positive de l'individu et croire que la bonne volonté et les compétences de l'individu suffisent à ne pas être déçu. Il s'agit là d'une confiance aveugle (Achrol, 1997, cité par Wicks et al, 1999) qui nie les risques notamment en cas d'interdépendance et qui peut mener rapidement à la déception, faisant renoncer à la vision positive de l'individu. La deuxième façon de se rassurer face au risque consiste, dans le cas où l'individu est vu comme opportuniste et aliéné à ses intérêts, à envisager la confiance comme l'attente qu'un individu ne va pas s'engager dans un comportement opportuniste malgré les motivations qu'il peut avoir, et ce, par logique calculatoire ou par effet de dissuasion. C'est sur ce postulat que se sont développées la plupart des théories cherchant à résoudre des situations où un individu doit agir dans l'intérêt d'un autre, qui lui délègue une partie de son pouvoir de décision, avec une approche purement disciplinaire de la performance et une optimisation du contrôle par le contrat (par exemple : Jensen et Meckling, 1976). La confiance est initialement apparue en gestion comme solution de dépit face aux limites de la rationalisation mais elle s'y développe désormais sur l'argument d'efficacité économique comme le meilleur moyen pour l'organisation d'atteindre les objectifs fixés (Bornarel, 2004). Les relations de confiance sont valorisées par des jeux subtils de management de sorte que le coût de la non confiance devient trop élevé, même si les individus ne sont pas considérés comme influencés par des motivations positives ou morales (Cohen et Dienhart, 2013). Cette combinaison d'un environnement favorable à la confiance et d'une vision négative de l'individu entraîne une confiance paradoxale souvent rencontrée dans l'entreprise moderne qui peut aisément être considérée comme de la manipulation et de l'hypocrisie (Bornarel, 2004).

Les approches de la confiance présentées nient soit la vision positive de l'individu, soit celle du risque, soit les deux. Et si combiner les deux était possible ? C'est la configuration correspondant à la confiance idéale dans la typologie proposée par Bornarel (2007). Elle associe un contexte d'indépendance et d'incertitude à l'hypothèse que l'homme agit en fonction d'intérêts jugés supérieurs aux siens, en prenant à la fois en compte ses intérêts et ceux des autres, ce qui s'inscrit dans la théorie du stewardship (Davis et al., 1997) et l'approche opale des entreprises (Laloux, 2014). Si Bornarel ne développe pas les façons d'atteindre cette confiance idéale, d'autres chercheurs donnent des pistes. Das et Teng (1998) repris par Fenneteau et Naro (2005) invitent à la confidence, une confiance mêlée de certitude qui intègre le risque en ayant la conviction (et non pas l'espoir) que ce risque n'est pas un danger. Castelfranchi et Falcone (2000), quant à eux, développent la notion de confiance globale qui ne porte pas que sur l'individu 
mais sur l'ensemble des facteurs de risques contextuels, internes et externes, en postulant que l'individu passe à l'action de faire confiance à la suite de l'évaluation de l'ensemble des facteurs, ce qui correspond à une vision positive prudente (Wicks et al., 1999). En associant ces approches, la confiance peut se traduire par la certitude que les objectifs seront atteints grâce à la combinaison de la croyance qu'autrui a l'intention de réaliser l'action, qu'il a les compétences pour le faire et qu'il fera effectivement ce qu'il faut pour y parvenir d'une part, et grâce à des moyens de prendre en compte les facteurs de risques internes et externes d'autre part. Les risques internes peuvent être liés à des biais cognitifs, une mauvaise évaluation du niveau de compétences, d'engagement, de volonté et de persévérance de l'individu, à une rationalité limitée, à des habitudes sociales ou conventions, ou à une défaillance de l'individu. Les facteurs externes incluent toutes les interférences et entraves éventuelles non liées à l'individu. Cette approche de la confiance se démarque à la fois de la vision opportuniste de l'individu et de la recherche de maitrise du risque puisqu'elle combine une vision positive de l'individu et une confiance organisationnelle qui intègre le risque. Nous l'appellerons confiance assurée. La question qui se pose par rapport aux entreprises qui se disent libérées est de savoir si la vision a priori de l'individu est positive ou négative d'une part, et si le risque lié au contexte d'incertitude et d'interdépendance dans lequel elles évoluent est nié, maîtrisé ou intégré.

\section{Tableau 1 : Différentes acceptions de la confiance}

\begin{tabular}{|c|c|}
\hline & Définition \\
\hline Trust & $\begin{array}{l}\text { Ensemble d'attitudes mentales qui caractérisent l'esprit de l'agent } \\
\text { X qui veut déléguer (core trust), et décide qu'un autre agent Y } \\
\text { fasse l'action (reliance trust) (Castelfranchi et Falcone, 2001). } \\
\text { Attente positive relative à l'intention et aux compétences d'un } \\
\text { autre menant à se fier à lui/elle dans une situation à risque (Das } \\
\text { et Teng, 1998). }\end{array}$ \\
\hline Global trust & $\begin{array}{c}\text { Combinaison de l'évaluation d'un individu Y à qui un agent } \mathrm{X} \\
\text { veut déléguer (core trust) et de celle des conditions } \\
\text { environnementales (environmental or situational trust) menant X } \\
\text { à l'action de déléguer à Y. } \\
\text { (Castelfranchi et Falcone, 2001) }\end{array}$ \\
\hline Confidence & $\begin{array}{l}\text { Certitude que le partenaire coopérera de manière satisfaisante, } \\
\text { sentiment de sécurité par rapport au comportement du partenaire } \\
\text { créé par la complémentarité entre la confiance (trust) accordée } \\
\text { au partenaire et le contrôle exercé sur celui-ci (Das et Teng, } \\
\text { 1998). }\end{array}$ \\
\hline
\end{tabular}




\begin{tabular}{|c|c|}
\hline $\begin{array}{l}\text { Confiance- } \\
\text { espoir }\end{array}$ & $\begin{array}{l}\text { Croyance dans le bon état d'esprit et les compétences d'autrui } \\
\text { pour réaliser une action dans une situation comportant un risque } \\
\text { non maîtrisé. }\end{array}$ \\
\hline \multirow{3}{*}{$\begin{array}{l}\text { Confiance } \\
\text { assurée }\end{array}$} & $\begin{array}{l}\text { Certitude que les objectifs seront atteints grâce à la combinaison } \\
\qquad \text { de : }\end{array}$ \\
\hline & $\begin{array}{l}\text { 1/ la croyance qu'autrui a l'intention de réaliser l'action, qu'il a } \\
\text { les compétences pour le faire et qu'il fera effectivement ce qu'il } \\
\text { faut pour y parvenir ; }\end{array}$ \\
\hline & $\begin{array}{l}\text { 2/ des moyens de prendre en compte les facteurs de risques liés } \\
\text { aux individus et aux conditions externes. }\end{array}$ \\
\hline
\end{tabular}

\subsection{Entre contrôles formels ou sociaux coercitifs et contrôles coopératifs}

Le contrôle organisationnel est l'influence sur les comportements des individus en vue d'atteindre des objectifs organisationnels, avec un double sens de vérification et de maîtrise. Il s'appuie sur deux types de mécanismes de contrôles, formels et sociaux (Tessier et Otley, 2012). Nous utiliserons le singulier pour le contrôle en général, et le pluriel pour se référer aux mécanismes de contrôle. Les contrôles formels sont constitués de mécanismes de contrôle des résultats d'une part, et de contrôle des comportements d'autre part (output control et behaviour control, Ouchi, 1980), ces derniers se déclinant de différentes manières. Les contrôles des résultats correspondent à la mesure d'indicateurs financiers (profits, ventes etc.) ou non financiers comme la qualité (Alvesson et Kärreman, 2004). Les contrôles techniques (Tessier et Otley, 2012) ou technocratiques (Alvesson et Kärreman, 2004) indiquent comment les tâches doivent ou ne doivent pas être réalisées et comment les individus et les groupes doivent ou ne doivent pas être organisés. Ils sont basés sur des règles, des procédures et des standards (Alvesson et Kärreman, 2004 ; Malmi et Brown, 2008 ; Tessier et Otley, 2012). Les contrôles administratifs (Malmi et Brown, 2008) ou bureaucratiques (Alvesson et Kärreman, 2004) dirigent les comportements des salariés à travers la façon dont l'organisation est conçue et structurée et la définition de qui est responsable par rapport à qui. Les contrôles cybernétiques correspondent à la définition de standards de performance à atteindre, à la mesure des activités correspondantes, à la comparaison des résultats avec les standards, à l'analyse de la variance et à la capacité de modifier le système en fonction (Malmi et Brown, 2008). Les mécanismes de contrôle formels, qui ont connu leur essor dans les organisations tayloriennes puis bureaucratiques, sont devenus inappropriés ou insuffisants dans un contexte d'agilité parce qu'ils ne sont plus un gage d'atteinte des objectifs fixés par l'organisation. Les situations gagnant en complexité, il devient impossible ou non viable économiquement de recourir à des règles, objectifs, procédures ou règlements qui spécifient les schémas de comportements attendus, la façon dont les tâches doivent être réalisées et dont les individus doivent s'organiser pour que les objectifs soient atteints (Leifer et Mills, 
1996). Se fier à des indicateurs de performance comme le chiffre d'affaires, la marge ou la qualité, en se concentrant sur les résultats mesurables, peut même être contreproductif parce qu'ils ne focalisent l'attention que sur les objectifs évalués (Ouchi, 1980).

L'impossibilité de convertir en indicateurs mesurables tous les objectifs et processus d'une organisation a entraîné l'apparition de nouveaux mécanismes de contrôle, qui font appel aux éléments émotionnels, non rationnels, affectifs des employés. Si les contrôles formels sont utilisés pour faire face a posteriori à la divergence d'intérêt entre les individus et le collectif, problème majeur de la coopération, si la régulation par le marché et par la bureaucratie ne suffit plus, alors une solution alternative vise à réduire cette divergence a priori en faisant en sorte que les individus partagent les objectifs de l'organisation grâce à un processus de socialisation (Ouchi, 1980). Ces contrôles informels (Tessier et Otley, 2012), culturels, sociaux correspondent à l'emploi de normes et valeurs organisationnelles pour encourager les comportements désirés. Ce contrôle culturel peut avoir lieu au moment du recrutement (Khodyakov, 2007 ; Malmi et Brown, 2008) pour choisir des individus qui partagent les normes et valeurs de l'organisation. Il peut avoir lieu après le recrutement, pour faire en sorte que les individus en viennent à intégrer les normes et valeurs (Malmi et Brown, 2008). Cela passe par exemple par la socialisation, des interactions, mais aussi par les messages véhiculés par les procédures (comme les évaluations) et le type de résultats valorisés (par exemple résultats financiers ou satisfaction clients) qui indiquent ce qui est bien, bon, souhaitable ou non (Alvesson et Kärreman, 2004). Selon les comportements et résultats valorisés, l'organisation influence les sources de motivation des individus et par là même la façon dont ils construisent leur identité, puisque celle-ci est liée à l'estime de soi et au besoin de se sentir en sécurité, et donc de correspondre à ce qui est attendu (ibid.). La culture organisationnelle devient un élément clé. En persuadant les individus d'adopter certaines valeurs, normes et idées sur ce qui est bien, important, louable en termes de travail et de vie organisationnelle, les organisations ont recours à un contrôle socio-idéologique (Alvesson et Kärreman, 2004) pour influer sur leurs comportements.

Chacun des types de contrôles formels et sociaux peut être utilisé dans une visée de diagnostic ou d'interactivité, pour habiliter ou contraindre, et dans une perspective de récompenses ou de punitions, et ce selon les intentions managériales (Tessier et Otley, 2012). Cette conception des systèmes de contrôle invite à sortir d'une logique duale et à dépasser le clivage entre bons et mauvais contrôles ou la question de savoir si les contrôles sociaux sont préférables aux contrôles formels. Il s'agit d'envisager l'ensemble des utilisations des contrôles dans une complémentarité qui permet de gérer les tensions entre liberté et contrainte, entre responsabilisation (empowerment) et responsabilité (accountability), entre direction descendante (top down) et créativité ascendante (bottom up), entre expérimentation et efficacité, ces tensions devenant créatrices de nouvelles possibilités (ibid.). La perception positive, neutre ou négative des contrôles par les 
salariés est une réponse émotionnelle face aux contrôles qui dépend non pas des contrôles eux-mêmes mais de la façon dont les contrôles sont présentés, des expériences précédentes du contrôle des salariés et des caractéristiques individuelles des salariés (environnement socioculturel, personnalité, etc.). Les contrôles ne sont donc pas intrinsèquement bons ou mauvais mais dépendent de l'intention dans laquelle ils sont utilisés et de la perception qu'en ont les salariés. Das et Teng (1998) postulent que les contrôles peuvent être utilisés par les parties dans leur intérêt commun. Il s'inscrivent alors dans une logique de coopération et de protection contre la domination (Khodyakov, 2007) et non dans une logique de maîtrise (Fenneteau et Naro, 2005). Reste à savoir quelle est l'intention des managers et la perception qu'ont les salariés des contrôles dans les entreprises libérées.

\subsection{Contrôles coopératifs et confiance idéale: une supplémentation créatrice d'assurance}

Dans l'approche économique classique dans laquelle l'individu est a priori opportuniste et aliéné à ses intérêts, la confiance dans l'individu n'a pas de place et le risque doit être maîtrisé au maximum. L'organisation scientifique du travail n'étant plus adaptée à l'incertitude et à l'interdépendance du contexte actuel, la confiance peut être utilisée comme un contrôle social lorsqu'elle est perçue comme plus efficace que d'autres types de contrôles (Bornarel, 2004). La complémentarité entre confiance et contrôle sert dans ce cas à renforcer le contrôle. Le rejet de ce qui peut être perçu comme une manipulation peut inciter à adopter une confiance totale par réaction opposée, rejetant tout contrôle (Getz, 2009), avec de forts risques d'être déçu puisque les risques sont niés. Une troisième voie est possible : il s'agit d'adopter une approche de la confiance qui repose sur une vision positive de l'individu, de sa bonne volonté et de ses compétences, et qui intègre le risque dans les pratiques au lieu de tenter de le maîtriser ou de le braver (Das et Teng, 1998). Dans cette logique, il devient possible d'envisager que les contrôles puissent être utilisés de manière coopérative (Khodyakov, 2007) pour intégrer différents risques non liés à un éventuel opportunisme mais à d'autres facteurs internes et externes (Castelfranchi et Falcone, 2000) et ainsi supplémenter la confiance en l'autre pour arriver à une confiance assurée (la confidence pour Das et Teng, 1998).

La vision positive de l'individu et la coopération n'étant pas la norme sociale actuelle, des cadres peuvent être nécessaires pour perdre les habitudes acquises si l'on postule que les individus peuvent agir dans un intérêt commun. Cette nécessité peut également être envisagée sous un angle biologique en considérant que l'évolution génétique de l'individu, doté depuis le paléolithique de pulsions de domination pour survivre dans un environnement hostile, n'a pas (encore) suivi l'évolution sociale permise par la plasticité cérébrale (Dupagne, 2012, ch. 6). Aussi, l'homme a besoin de cadres limitant les risques de recours à la domination pour que sa capacité de relations basées sur la confiance et l'empathie s'exprime 
pleinement. Par ailleurs, la rationalité limitée peut entraîner la personne la plus fiable et la mieux intentionnée à partager par erreur une information inexacte, invalide ou périmée (McEvily et al., 2003). Ainsi, les mécanismes de contrôle, s'ils sont bien utilisés, peuvent aider à construire la confiance mutuelle grâce à un processus d'évaluation objective au lieu d'une impression subjective (Das et Teng, 1998).

Pour que des contrôles puissent servir la confiance, il faut une base de vision positive et une volonté de la développer : le contrôle ne peut en effet pas créer la confiance mais il peut la renforcer. Cette base peut venir d'une inclination naturelle (Rotter, 1967), d'une influence culturelle plus ou moins favorable, ou d'expériences antérieures de confiance, entre les parties elles-mêmes, ou entre les parties et des tiers (McEvily et al. 2003). Dans le cadre des alliances stratégiques, Das et Teng (1998) ont identifié trois façons dont les contrôles peuvent supplémenter la confiance "trust» pour développer une confiance "confidence » : des processus de décision participatifs pour prendre les décisions en commun et pouvoir faire évoluer les objectifs au fur et à mesure en développant une meilleure connaissance mutuelle et un meilleur consensus, des spécificités structurelles qui limitent les contrôles formels et, enfin, le développement d'une culture commune. Ces trois aspects se retrouvent dans l'étude de Khodyakov sur l'orchestre Orpheus (2007), auxquels s'ajoutent une sélection à l'entrée qui porte non seulement sur les compétences mais aussi sur des caractéristiques interpersonnelles, des contrôles multidirectionnels qui permettent à chacun d'avoir des informations précises et fiables sur les plans du management et d'influencer les décisions, des contrôles des comportements au service de la coopération, ainsi qu'une conscience de soi à travers une attention de chacun portée à son propre comportement.

Les exemples phares d'entreprises libérées mis en avant par les discours managériaux s'appuient explicitement sur la vision positive de l'individu de la théorie Y de Douglas Mc Gregor (Getz, 2013, p.18), de même que l'approche opale des organisations repose sur un a priori positif de l'autre (Laloux, 2014). À cette vision s'ajoute l'importance de considérer les problèmes et échecs comme source d'apprentissage, partant du fait que tout le monde apprend en permanence et qu'échanger sur ses erreurs au lieu de les cacher permet de se développer en tant qu'individu et qu'organisation (Laloux, 2014, p.230-231). " Si vous n'avez pas fait la moindre erreur, c'est que vous n'avez pas pris suffisamment de risque » dit Bill Gore (cité par Getz, 2013, p.168). Il ne s'agit pas de nier les risques mais de ne plus en avoir peur (Laloux, 2014) et de prendre les décisions selon le principe de la ligne de flottaison (Getz 2013), d'intégrer les risques potentiels pour l'ensemble des parties concernées à travers un processus de consultation ou de concertation adapté à la situation, d'assumer la responsabilité de ses choix et de réajuster autant que nécessaire. Les récits managériaux font état de principes ou règles du jeu propres à chaque entreprise libérée qui apparaissent de manière organique pour "nourrir l'impératif de coordination qui reste une réalité » et s'inscrivent au service d'une vision partagée (Getz, 2013, p.260). Ainsi, ces entreprises allient un 
a priori positif de l'individu et une intégration du risque dans un contexte d'incertitude et d'interdépendance, et ce grâce à des mécanismes de coordination qui s'inscrivent dans une perspective de coopération au service d'une vision commune. Cette combinaison fait des entreprises «libérées» ou «opale » un terreau propice à une articulation entre confiance et contrôle qui permet de développer une confiance assurée.

Cette articulation confiance-contrôle au service de la confiance n'a pas été testée empiriquement dans les entreprises libérées. Nous présentons dans la partie suivante la recherche que nous avons conduite pour explorer la façon dont des salariés et managers vivent l'articulation entre confiance et contrôle dans une organisation qui s'inscrit dans ce courant bien qu'elle n'utilise pas ce terme.

\section{3. ÉTUDE DE CAS : LA CONFIANCE COMME PRINCIPE ORGANISATIONNEL}

\subsection{Choix d'une équipe de l'entreprise Hervé Thermique}

Notre problématique se concentrant sur l'articulation entre confiance et contrôle, nous avons cherché, pour l'étude de cas, une organisation qui évolue dans un contexte de forte interdépendance et qui prône la confiance comme étant au cœur de son management, tout en ayant recours à des mécanismes de contrôle forts. Hervé Thermique ${ }^{3}$ répondait à ces critères. Cette entreprise familiale du BTP affiche que «la conviction présidant à cette organisation innovante est qu'un homme ne donne le meilleur de lui-même qu'à partir du moment où il prend conscience qu'on lui fait confiance ${ }^{4} »$, tout en revendiquant une hiérarchie à quatre niveaux et des mécanismes de régulation, tels que des réseaux fonctionnels transverses. Le groupe Hervé articule son fonctionnement autour de trois modalités : déhiérarchisation du pouvoir, intra-entrepreneuriat et organisation en réseaux. La première consiste à supprimer le pouvoir de domination associé à la hiérarchie et à le remplacer par le pouvoir de responsabilisation et l'obligation morale de chaque salarié. La seconde implique de permettre à chacun d'être son propre chef en déterminant non seulement les moyens mais aussi les fins qu'il poursuit. Enfin, la cohérence de l'organisation repose sur la libre association de ses membres à travers des coopérations libres et imprévisibles entre réseaux (Hervé et Brière, 2012, p.9-10). Au-delà d'une déclaration d'intention sur un site internet, la vision de l'organisation qui se veut « démocratique », " participative », « 2.0 », fait l'objet à la fois d'une expérimentation depuis quatre décennies et d'une

3 Les auteurs remercient Hervé Thermique d'avoir permis la réalisation de cette recherche.

4 http://www.groupeherve.com/organisation-participative/intra-entreprenariat, consulté le 3 mars 2014 
rationalisation et conceptualisation qui se sont traduites par la publication de trois ouvrages (Hervé et al., 2007 ; Hervé et Brière, 2011 ; Hervé, 2015).

Nous avons choisi de nous focaliser sur les représentations au niveau individuel pour comprendre comment les salariés perçoivent l'articulation entre mécanismes de contrôle et confiance dans la pratique. Le tableau 2 présente les caractéristiques de l'échantillon retenu tandis que l'encadré présente la méthodologie de l'étude et la figure 1 un exemple de carte cognitive issue de l'étude de cas.

Tableau 2 : Caractéristiques de l'échantillon cartographié

\begin{tabular}{|c|c|c|c|}
\hline Fonction & $\begin{array}{c}\text { Années } \\
\text { d'ancienneté }\end{array}$ & Tranche d'âge & $\begin{array}{c}\text { Durée de } \\
\text { l'entretien }\end{array}$ \\
\hline Eesponsable de chantier & 28 & $>50$ & $1 \mathrm{~h}$ \\
\hline Responsable de chantier & $\begin{array}{c}2(+4 \\
\text { d'intérim) }\end{array}$ & $<30$ & $45 \mathrm{~min}$ \\
\hline $\begin{array}{c}8(+3 \\
\text { d'intérim })\end{array}$ & $30-40$ & $1 \mathrm{~h} 15$ \\
\hline $\begin{array}{c}5(+17 \\
\text { d'intérim) }\end{array}$ & $30-40$ & $45 \mathrm{~min}$ \\
\hline Chef de projet & 20 & $40-50$ & $1 \mathrm{~h}$ \\
\hline Assistante de gestion & 12 & $30-40$ & $45 \mathrm{~min}$ \\
\hline $\begin{array}{c}\text { Futur manager d'activité } \\
\text { (en formation dans } \\
\text { l'équipe) }\end{array}$ & 7 & $30-40$ & $1 \mathrm{~h} 30$ \\
\hline $\begin{array}{c}\text { Ancien manager d'activité } \\
\text { de l'équipe }\end{array}$ & 35 & retraité & $45 \mathrm{~min}$ \\
\hline $\begin{array}{c}\text { Manager d'activité (en } \\
\text { exercice) }\end{array}$ & 16 & $40-50$ & $1 \mathrm{~h} 30$ \\
\hline $\begin{array}{c}\text { Manager de territoire } \\
\text { Eantier }\end{array}$ & 15 & $30-40$ & $1 \mathrm{~h} 45$ \\
\hline
\end{tabular}




\section{Figure 1 : Exemple de carte cognitive (entretien A)}

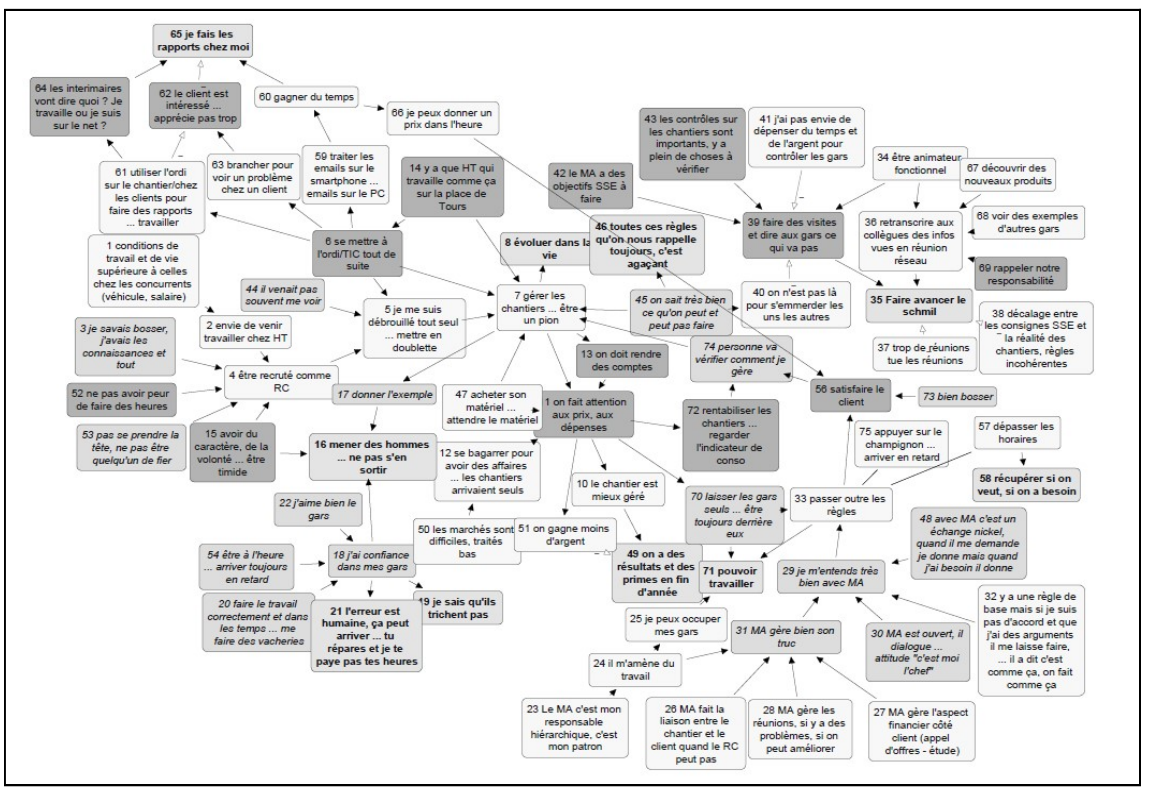

\section{Méthodologie}

Une recherche empirique exploratoire a été réalisée au sein d'Hervé Thermique, entreprise familiale du BTP comptant 1950 salariés. Cette organisation met en avant la confiance, l'autonomie et la responsabilisation de chacun de ses employés et a mis en place des structures organisationnelles pour y parvenir. Nous nous sommes concentrés sur une équipe, que nous avons observée en situation (chantier, bureau, réunion) avant d'en rencontrer les membres et leurs managers pour dix entretiens libres individuels (cf. tableau 2). Ce choix reflète notre volonté de privilégier une étude en profondeur au niveau des salariés pour faire émerger leurs représentations $\mathrm{du}$ fonctionnement de l'organisation et de la relation entre confiance et contrôle. Pour augmenter la validité interne de l'étude, nous avons construit un guide d'entretien favorisant les questions ouvertes afin de collecter les représentations des salariés sur la façon de travailler ensemble au sein de l'organisation. Les répondants n'ont pas été interrogés directement sur le contrôle et la confiance de sorte que l'occurrence de ces notions est spontanée et non assistée. Retranscrits intégralement, les entretiens ont été codés grâce à l'identification des variables influençantes, liens d'influence ${ }^{5}$ et liens influencés pour représenter les entretiens sous forme de cartes cognitives individuelles

$5 \rightarrow$ : les flèches à tête noire indiquent une influence positive, tandis que les flèches à tête blanche indiquent une influence négative 
(Allard-Poesi, 2007) à l'aide du logiciel Banxia Decision Explorer 3.3.1. Un double codage sur une partie des entretiens a permis d'améliorer la fiabilité. Pour faire émerger les représentations propres et privilégier la spontanéité, nous n'avons pas fait appel à des concepts définis au préalable et avons limité les fusions. Les cartes ont été analysées à partir des queues (concepts influençants), têtes (concepts influencés), concepts centraux, et de leur articulation. Les données issues de l'observation et de sources complémentaires ont été utilisées pour trianguler les données.

\subsection{De la confiance idéale à la confiance assurée}

La confiance interpersonnelle est apparue spontanément dans les représentations de neuf des dix personnes interrogées, faisant apparaître une croyance dans les bonnes intentions ainsi que dans les compétences, le sérieux et la fiabilité, aussi bien entre salariés que de la part des salariés à l'égard de leur manager et inversement. La majorité des items liés à ces dimensions apparaissent dans les queues et concepts centraux et sont donc des variables influençantes. Ces croyances qui servent de base à la confiance interpersonnelle sont complétées par une vision de l'organisation qui fait confiance à ses salariés en se souciant d'eux ("C'est une entreprise qu'a un certain respect pour ses employés, (...) y a une écoute, (...) on fait attention $\grave{a}$ toi $" \mathrm{~K} 41^{6}$ ), et en favorisant effectivement l'autonomie et la responsabilisation ("Ici on gère vraiment tout» G4; "J'avais une très grande autonomie dans ma fonction de technicien » $\mathrm{B} 3$ ). Cette vision de l'organisation est cohérente avec les intentions managériales affichées dans les ouvrages publiés par le fondateur (Hervé 2007, 2011, 2015).

Dans ces mêmes neuf cartes apparaissent dans les têtes des items liés à une confiance assurée, qui sont de deux ordres. Le premier aspect est une assurance sur l'avenir de l'entreprise, son développement, sa pérennité, à travers des variables comme "faire avancer le schmil" "(A35), "aider l'entreprise à vivre et à se développer" (B8), ou une "structure pérennisée, solide »(E49). Le deuxième aspect concerne la capacité à se sentir bien dans cet environnement et à s'y développer personnellement: "s'enrichir humainement et en compétences" (B75), «j'ai évolué intellectuellement, ça m'a enrichi »(F81).

L'étude des représentations dans lesquelles la confiance assurée est vécue fait apparaître la combinaison d'une grande confiance interpersonnelle avec un rapport au contrôle particulier. "On n'est pas "flicking", on n'est pas le grouillot de service, c'est ça qu'est agréable par rapport à d'autres sociétés »(E13). Les contrôles sont utilisés dans une logique de coopération et pour l'intérêt collectif, «dans un souci d'aide et d'analyse » (B50), dans une finalité de contribution au

6

Les lettres et chiffres font référence aux cartes et numéros des concepts auxquels renvoient les verbatims, en l'occurrence le concept $n^{\circ} 41$ de la carte de l'entretien K. 
bien commun. «Je suis là pour aider, mais s'il veut pas plus, je lui mets pas le couteau sous la gorge " (K58). "On n'est pas là pour s'emmerder les uns les autres (A40).

Non seulement les individus mentionnent particulièrement la confiance dans la bonne volonté et les compétences des autres, mais en plus ils considèrent les contrôles comme des cadres nécessaires pour bien travailler ensemble parce qu'ils ont conscience des facteurs de risques internes et externes ( $" y$ a des phénomènes de caractères » $\mathrm{B} 24$, "y peut toujours y avoir de l'abus ou des erreurs en défaveur de l'entreprise» B45 mais aussi «en défaveur des salariés» B46). Ces représentations n'empêchent pas les individus de mentionner que tout n'est pas évident au quotidien et que l'image n'est pas parfaite. Cependant, ces éléments reflètent alors une conscience des failles et limites à intégrer et d'évolutions à apporter plutôt qu'une remise en question du fonctionnement. La vision à la fois positive et prudente est illustrée dans la figure 1. La partie suivante présente la façon dont ces cadres nécessaires se mettent en œuvre au service de la confiance assurée.

Figure 1 : Une vision positive prudente (extrait de la carte B)

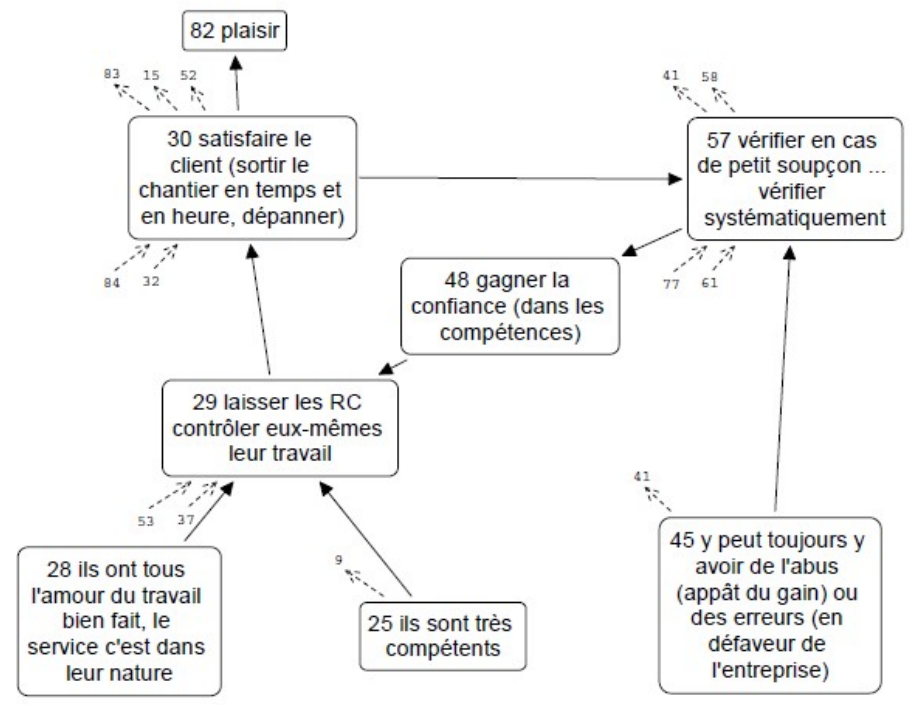

\subsection{Mise en œuvre des contrôles coopératifs}

Khodyakov (2007) avait identifié l'importance de la conscience de soi et de sa propre attitude pour que des contrôles puissent être vus comme coopératifs. De même, l'autodiscipline apparaît comme une des trois clés des entreprises libérées 
pour Getz (2013). Cette notion ressort chez Hervé Thermique dans l'importance de l'exemplarité des managers vis-à-vis de leurs équipes comme des responsables de chantier vis-à-vis des intérimaires : "C'est à nous de faire mieux qu'eux, enfin, pas mieux qu'eux mais être toujours à l'heure, etc. » (A17). "C'est à toi de tenir le fil droit » (K16). L'impact de l'exemplarité reflète l'existence de cercles vicieux comme vertueux: des attitudes négatives et incohérentes entraînent des comportements opportunistes tandis que des attitudes positives et cohérentes entraînent des comportements dignes de confiance (Wicks et al., 1999). L'importance accordée à la conscience de soi se voit également dans le parcours suivi par le futur manager d'activité, qui inclut des moyens de comprendre les réflexes et automatismes qu' [il peut] avoir (D21).

Cette attention portée à sa propre attitude s'ajoute à l'importance de l'autocontrôle, à savoir le suivi et la vérification de ses propres actions et résultats Les chefs de chantiers et managers doivent compléter un certain nombre d'outils informatiques pour suivre les résultats, qu'ils soient financiers (chiffre d'affaires, marge, rentabilité) ou non (satisfaction client). Cela leur permet de suivre [leurs] propres indicateurs et voir les sonnettes d'alarme (K68), et sont donc des moyens de prise de décision (Malmi et Brown, 2008). Ils peuvent apparaître comme des moyens de contrôle dans la mesure où ils servent aussi à rendre des comptes aux collègues et aux supérieurs, sauf s'ils ne sont pas utilisés dans une perspective de punition ou de récompense mais dans un intérêt commun, par exemple pour éviter les oublis (E37) ou faire avancer le travail des autres (E34). Le suivi par le supérieur vient en complément et non en usage principal, ce qui donne le sentiment d'être responsabilisé (E13), de [ne pas avoir] ce poids de la hiérarchie, bien qu'[ils aient leur] responsable quand même au-dessus. Ceci est gratifiant et agréable (E12), donne envie de rester dans l'entreprise (E16) et de prendre en charge les conséquences de ses propres erreurs (E15). Cette vigilance et ce soutien sont également assurés par des réseaux fonctionnels transverses qui ont été mis en place en complément de la structure opérationnelle et auxquels chaque employé peut participer, peu importe sa fonction opérationnelle.

Les réseaux transverses et les réunions des structures opérationnelles, par la façon dont ils sont organisés, permettent aux salariés d'influer sur les décisions et l'adaptation des règles de fonctionnement de l'organisation et des outils de suivi. "L'avantage, c'est que quand on a quelque chose qui descend, qui nous arrive, parce que souvent ça nous arrive, malgré tout, et qu'on peut le critiquer, qu'on peut l'améliorer, c'est quand même bien » (B33). Ces réseaux permettent notamment de veiller à ce que les outils mis en place soient toujours pertinents et utiles pour les utilisateurs eux-mêmes. Ainsi, les contrôles hiérarchiques des résultats sont limités par les spécificités structurelles, comme identifié par Das et Teng (1998). Les employés peuvent non seulement participer à la création des règles elles-mêmes, mais aussi les ajuster à chaque situation. Les règles sont un guide au service d'une finalité (rentabilité, satisfaction client, etc.) et liberté est donnée à chaque salarié d'adapter la règle lorsque cela est nécessaire pour mieux 
servir cette finalité. "Y a une règle de base mais si je suis pas d'accord et que j'ai des arguments, il me dit ben écoute, fais comme tu le sens»(A32). Si la règle apparaît comme l'objectif final, cela est agaçant (A46) voire démotivant (D69). En revanche lorsqu'ils sentent qu'ils participent à un projet global, qu'ils apportent à l'organisation, voire à la société, de façon plus large que des simples tâches exécutées, les salariés ont plaisir à travailler. «Le soutien il est là, quoi, j'veux dire, c'est quand on te fait prendre conscience de la globalité du projet » (K31). «Il y a une notion de plaisir, au-delà du fait qu'il faut apporter de l'argent à l'entreprise et tout ça » (B7). Ces éléments rejoignent l'importance de mécanismes de décision participatifs qui permettent une meilleure compréhension mutuelle identifiés par Das et Teng (1998).

La conscience de sa propre attitude, la prise de responsabilité de ses actions ou la participation aux décisions organisationnelles demandent des qualités qui sont intégrées dans les critères de recrutement et dans les processus d'intégration et d'évolution. Pour être recruté et durer dans le groupe, il faut à la fois un fort caractère et de l'humilité, une capacité à se remettre en question, une attention aux autres et le sens de l'intérêt collectif, ce qui est résumé par le manager de territoire et le manager groupe par " empathie, humilité, curiosité et transversalité » (C62), en plus de compétences techniques. Par exemple si un manager n'a pas les caractéristiques interpersonnelles qui permettent de tolérer suffisamment le risque, il peut être amené à rappeler excessivement les règles (D64) ce qui génère inquiétude (D63) et démotivation (D69), ce qui réduit l'investissement spontané (D67) et entraîne un cercle vicieux. Ceci rejoint la sélection à l'entrée à la fois sur les compétences et sur des caractéristiques interpersonnelles comme la capacité d'écoute, la volonté de prendre des initiatives, la volonté d'exprimer ses opinions personnelles ou encore la capacité à être responsable de ses décisions et actions et à accepter ses erreurs identifées par Khodyakov (2007) dans son étude de l'orchestre Orpheus. En effet, la confiance peut être augmentée par les contrôles mais ne peut pas être créée. Les contrôles à l'entrée permettent de s'assurer que les salariés ont un minimum de vision positive de l'autre a priori. Depuis quelques années, un programme d'intégration a été mis en place pour chaque salarié qui intègre l'organisation ou change de poste. Ce programme vise à ce que les salariés intègrent le fonctionnement (C78) et partagent l'objectif principal de l'organisation (Ouchi, 1980): "prendre conscience qu'ils sont intra-entrepreneurs, chefs d'entreprise dans une entreprise " (C37). Khodyakov (2007) parle de même de processus de socialisation et de nécessité de s'engager pour le fonctionnement sans chef d'orchestre. Ainsi, des contrôles sociaux sont à l'œuvre pour favoriser la confiance. 


\section{CONCLUSION : UNE FAÇON D'ENVISAGER L'ORGANISATION ET LES RELATIONS}

Les entreprises peuvent-elles faire confiance à la confiance ? Pour que cela soit possible, il faut s'affranchir de deux conceptions pièges de la confiance : "Faites ce que vous voulez, tout ira bien! 》 et "T'as bien compris, j'te fais confiance, hein? ». La première attitude correspond à une confiance en l'autre aveugle, trop simpliste pour le contexte d'interdépendance et de multiples risques actuels. La deuxième posture est de la méfiance déguisée en confiance, qui utilise cette dernière comme la stratégie la plus efficace pour limiter les risques alors que la vision de l'individu lui-même est négative. Dans ce cas, la confiance, qualifiée de confiance paradoxale, est au service du contrôle et peut aisément être assimilée à de la manipulation. Des contrôles coercitifs existent alors souvent tout en étant minimisés voire déguisés. Faire confiance à la confiance requiert un changement d'attitude double par rapport à la norme actuelle, en adoptant une vision positive de l'individu et un rapport au risque qui ne cherche pas à l'éviter ou le maîtriser mais le prend en compte et s'adapte constamment. Ce changement d'attitude permet d'envisager que des contrôles soient coopératifs, en étant conçus et mis en œuvre de manière participative et conjointe par les parties de façon à servir un intérêt commun. Utilisés de la sorte, les contrôles viennent servir la confiance non seulement dans l'autre mais aussi dans l'organisation et dans l'avenir en développant l'assurance que quoi qu'il arrive, il sera possible de s'y ajuster. Le tableau suivant récapitule ces différentes articulations entre contrôle(s) et confiance.

Tableau 3 : Articulation confiance-contrôle

\begin{tabular}{|c|c|c|}
\hline & $\begin{array}{c}\text { Maîtrise ou déni du } \\
\text { risque }\end{array}$ & Intégration du risque \\
\hline $\begin{array}{c}\text { Vision négative } \\
\text { de l'individu }\end{array}$ & Confiance & $\begin{array}{c}\text { Confiance organisationnelle } \\
+ \text { contrôles coercitifs } \\
\Rightarrow \text { Contrôle }\end{array}$ \\
\hline $\begin{array}{c}\text { Contrôle } \\
\text { de l'indion positive }\end{array}$ & $\begin{array}{c}\text { Confiance } \\
\text { espoir aveugle }\end{array}$ & $\begin{array}{c}\text { Confiance organisationnelle } \\
+ \text { contrôles coopératifs } \\
\text { Contrôles }\end{array}$ \\
\hline
\end{tabular}

Notre étude fait ressortir empiriquement la combinaison d'un a priori positif sur l'état d'esprit et les compétences des individus (confiance interpersonnelle teintée d'espoir) et d'un contexte d'incertitude et d'interdépendance, configuration favorable à la confiance idéale (Bornarel, 2007). Elle identifie l'ajout d'une prise 
en compte de facteurs de risques internes et externes (Castelfranchi et Falcone, 2000) à travers des contrôles coopératifs (Khodyakov, 2007) qui servent de variable modératrice pour atteindre une confiance élargie pleine d'assurance (la confidence de Das et Teng, 1998). Das et Teng (1998) ont émis l'hypothèse que ce ne sont pas tant les mécanismes de contrôle eux-mêmes qui entachent ou enrichissent la confiance, mais leur forme, et Tessier et Otley (2012) ont émis l'hypothèse que les contrôles ne sont intrinsèquement ni bons ni mauvais mais que leur usage peut être bon ou mauvais selon l'intention managériale et la perception des salariés. Les résultats de notre étude s'inscrivent dans cette lignée et indiquent que ce n'est pas le caractère formel ou social des mécanismes qui importe, comme le supposaient Das et Teng, mais plutôt la logique dans laquelle ils s'inscrivent, soit de domination et coercition, soit de coopération.

Au-delà de ces apports, cette recherche exploratoire concentrée sur une équipe unique mériterait d'être élargie pour identifier si toutes les entreprises libérées sont le terrain de la confiance assurée. Elle gagnerait également à être complétée pour détailler l'étude des mécanismes de contrôle à l'œuvre, les modalités des contrôles coopératifs et la façon dont ils s'articulent avec la confiance dans un processus évolutif. Comment permettent-ils de développer une vision positive de l'autre et du risque ? Celle-ci peut-elle être décrétée, imposée, développée ? Chez tout le monde ou existe-t-il des facteurs de contingence ? Des liens avec les études sur la responsabilisation et l'empowerment et des approches de leadership associées, comme le servant leadership, et d'autres façons d'envisager l'organisation comme la stewardship theory, permettraient d'approfondir les conditions dans lesquelles la confiance peut être non pas un effet de mode ou de rhétorique ou une technique de manipulation mais une nouvelle façon d'envisager les organisations. Ainsi, alors que la plupart des théories et des pratiques managériales tiennent implicitement pour référence le panoptique et sa volonté de surveillance efficiente, véritable « événement dans l'histoire de la pensée » (Foucault, 1975), certes, mais qui érige le contrôle en mode essentiel de gouvernement des individus, les approches fondées sur la confiance semblent pouvoir constituer un paradigme alternatif. 


\section{BIBLIOGRAPHIE}

ALLARD-POESI F. (2007), Construction de l'objet de la recherche, in Thiétart, R.-A. Méthodes de recherche en management ( $3^{\mathrm{e}}$ édition.). Paris : Dunod, p. 34-56.

ALVESSON, M., \& KÄRREMAN, D. (2004), Interfaces of control. Technocratic and socio-ideological control in a global management consultancy firm. Accounting, Organizations and Society, vol. 29, n³-4, p. 423-444.

BORNAREL, F. (2004), La confiance comme mode de contrôle social. L'exemple des cabinets de conseil, Université de Nanterre - Paris X. 641p.

BORNAREL, F. (2007), La confiance comme instrument d'analyse de l'organisation, Revue française de gestion, vol. 33, n 175, p. 95-109.

CASTELFRANCHI, C., FALCONE, R. (2000), Trust and Control: A Dialectic Link, Applied Artificial Intelligence, vol. 14, $\mathrm{n}^{\circ}$ 8, p. 799-823.

COHEN M.A., DIENHART J. (2013), Moral and Amoral Conceptions of Trust, with an Application in Organizational Ethics, Journal of Business Ethics, vol 112, p.1-13

DAS, T. K., TENG B. S. (1998), Between Trust and Control: Developing Confidence in Partner Cooperation in Alliances, Academy of Management Review, vol. 23, n 3, p. 491-512.

DAVIS J.H., SCHOORMAN F. D., Donaldson L. (1997), Toward a stewardship theory, Academy oi Management Review, Vol. 22, No. 1, p. 20-47.

DUPAGNE, D. D. (2012), La revanche du rameur, Michel Lafon, 290p.

FENNETEAU, H., NARO, G. (2005), Contrôle et confiance dans l'entreprise virtuelle Illustrations logistiques, Revue Française de Gestion, vol. 31, $\mathrm{n}^{\circ} 156$, p. 203-219.

FOUCAULT, M. (1975), Surveiller et punir. Naissance de la prison, Gallimard.

GETZ, I. (2009), Liberating Leadership: How the initiative-freeing radical organisational form has been successfully adopted. California Management Review, vol. 51, n 4 , p. 32-58.

GETZ, I. CARNEY, B.-M. (2013), Liberté \& Cie, Quand la liberté des salariés fait le succès des entreprises, Ed. Flammarion, 421p. (2009 Freedom Inc., New York, Crown Business, trad. franç. 2012, Librairie Arthème Fayard)

GRATACAP, A., LE FLANCHEC, A., DE BOISSIEU, C., Oguchi, H. (2011), La confiance en gestion : un regard pluridisciplinaire. Bruxelles: De Boeck. $232 \mathrm{p}$.

HERVE, M., D'IRIBARNE, A., BOURGUINAT, E., Royal, S. (2007), De la pyramide aux réseaux : récits d'une expérience de démocratie participative. Paris: Éd. Autrement, 316 p. ;

HERVE, M., BRIERE, T. (2011), Le pouvoir au-delà du pouvoir : l'exigence de démocratie dans toute organisation. Paris: F. Bourin. 505 p. ;

HERVE, M. Une nouvelle ère, sortir de la culture du chef, (2015), Paris: F. Bourin, $229 \mathrm{p}$. 
INSTITUT MONTAIGNE, FINANCI'ELLES et VOUTCH (2014), Et $\boldsymbol{l a}$ confiance, bordel ? Faire le pari de la confiance en entreprise, Ed. Eyrolles, Paris, $244 \mathrm{p}$.

JENSEN, M. C., MECKLING W. H. (1976), Theory of the firm : managerial behavior, agency costs and ownership structure, Journal of Financial Economics, vol. 3, p. 305-360.

KHODYAKOV, D. M. (2007), The Complexity of Trust-Control Relationships in Creative Organizations: Insights From a Qualitative Analysis of a Conductorless Orchestra., Social Forces, vol. 86, n 1, p. 1-22.

LALOUX, F. (2014), Reinventing Organizations: A guide to creating Organizations inspired by the next stage of human consciousness, Ed.Nelson Parker, $382 \mathrm{p}$.

LEIFER, R., MILLS, P. K. (1996), An Information Processing Approach for Deciding Upon Control Strategies and Reducing Control Loss in Emerging Organizations, Journal of Management, vol. 22, $\mathrm{n}^{\circ}$ 1, p. 113-137.

MALMI, T., \& BROWN, D. A. (2008), Management control systems as a package-Opportunities, challenges and research directions, Management Accounting Research, vol.19, $\mathrm{n}^{\circ} 4$, p. 287-300

MCEVILY, B., PERRONE, V., \& ZAHEER, A. (2003), Trust as an Organizing Principle. Organization Science, vol.14, n ${ }^{\circ}$, p. 91-103

OUCHI, W. G. (1980), «Markets, Bureaucracies, and Clans », Administrative Science Quarterly, vol. 25, $\mathrm{n}^{\circ}$ 1, p. 129-141.

ROTTER, J. B. (1967). A new scale for the measurement of interpersonal trust. Journal of Personality, 35(4): 651-665

TESSIER, S., OTLEY, D. (2012), A conceptual development of Simons' Levers of Control framework, Management Accounting Research, vol. 23, $\mathrm{n}^{\circ} 3$, p. 171-185.

WICKS, A. C., BERMAN, S. L., JONES, T. M. (1999), The Structure of Optimal Trust: Moral and Strategic Implications, Academy of Management Review, vol. 24, n 1, p. 99-116. 